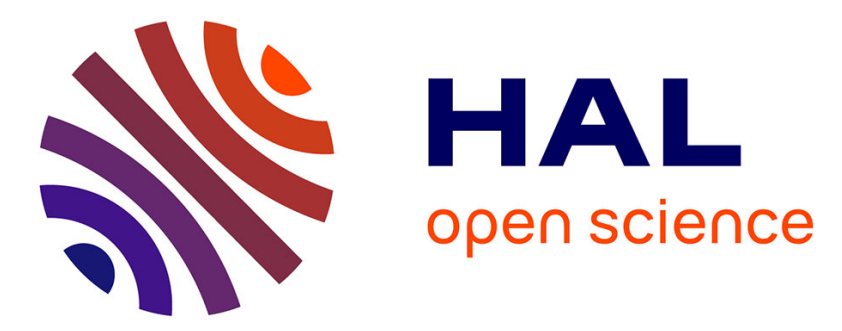

\title{
Spatial Data Focusing: An Alternative to Beamforming for Geocasting Scenarios
}

Julien Sarrazin, Michael Odhiambo, Sidney Golstein, Philippe de Doncker, François Horlin

\section{- To cite this version:}

Julien Sarrazin, Michael Odhiambo, Sidney Golstein, Philippe de Doncker, François Horlin. Spatial Data Focusing: An Alternative to Beamforming for Geocasting Scenarios. Conference USNC-URSI 2018, Jul 2018, Boston, United States. 10.1109/USNC-URSI.2018.8602761 • hal-02870089

\section{HAL Id: hal-02870089 \\ https://hal.sorbonne-universite.fr/hal-02870089}

Submitted on 16 Jun 2020

HAL is a multi-disciplinary open access archive for the deposit and dissemination of scientific research documents, whether they are published or not. The documents may come from teaching and research institutions in France or abroad, or from public or private research centers.
L'archive ouverte pluridisciplinaire HAL, est destinée au dépôt et à la diffusion de documents scientifiques de niveau recherche, publiés ou non, émanant des établissements d'enseignement et de recherche français ou étrangers, des laboratoires publics ou privés. 


\section{Spatial Data Focusing: an alternative to Beamforming for geocasting scenarios}

\author{
Julien Sarrazin, Michael Odhiambo \\ Sorbonne Université \\ Laboratory of Electronics and Electromagnetism, L2E \\ F-75005 Paris, France \\ julien.sarrazin@upmc.fr
}

\author{
Sidney Golstein, Philippe De Doncker, François Horlin \\ Université Libre de Bruxelles (ULB) \\ Av. Roosevelt 50 \\ 1050 Brussels, Belgium
}

\begin{abstract}
An alternative scenario is introduced in order to overcome antenna array's beamwidth limitations due to finite aperture size. The proposed approach aims to focus the transmitted data rather than the transmitted power. This scheme enables wireless broadcast of information to specific spatial locations, using fewer antenna elements compared to classical beamforming techniques. It is shown indeed with numerical examples that focusing the data is spatially more selective than focusing the power. In particular, we show that a linear 2-antenna array using spatial data focusing can exhibit similar beamwidths to a linear 7-antenna array using power focusing.
\end{abstract}

\section{INTRODUCTION}

Associating wireless information to certain physical locations is an interesting feature that many applications can benefit from. This capability, known as geocasting, enables to tag a real physical location by wirelessly transmitting data that are only decodable within desired delimited areas. Thus, users can receive information related to the place where they are without being localized, which respects their privacy, and which enables geocasting in areas where no positioning system is available, e.g., indoor environments.

To do so, base stations have to exhibit spatial focusing capabilities. Antenna arrays can be used for that purpose and beamforming techniques applied so that base stations can radiate signals toward particular directions only. However, the radiated beamwidth is determined by the electrical size of the array and achieving narrow beams often leads to unpractical large arrays. To overcome this limitation, this paper introduces a Spatial Data Focusing (SDF) scheme that aims to focus the radiated data rather than the power as classicaly done in beamforming [1]. In doing so, narrower beams can be obtained for a given number of antennas. Section II reviews and highlights the limitations of classical arrays in terms of beamwidth within which the transmitted data can be correctly retrieved. Section III introduces the SDF scheme and shows simulation results. Conclusions are drawn in section IV .

\section{Power FOcUsing ANGUlaR Limitation IN ANTENNA ARRAYS}

The linear array under consideration, shown in Fig. 1 . is composed of $N$ elements uniformly fed. Depending on the user's angular location $\theta$, the received power will vary according to the array factor and hence the received bit error rate (BER) too. While the beamwidth is classically defined at half-power, considering the geocasting application, we define the beamwidth as the beam inside which the received BER is below a certain threshold.

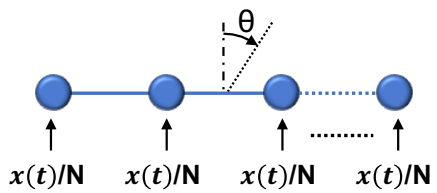

Fig. 1. $N$-antenna array.

To illustrate the focusing performance of this array, let us consider QPSK and 16-QAM modulations. Their BER can be given analytically by (1) and (2), respectively [2]:

$$
\begin{gathered}
P_{b}=Q\left(\sqrt{2 \frac{\gamma(\theta)}{k}}\right) \\
P_{b}=\frac{3}{4} Q\left(\sqrt{\frac{4}{5} \frac{\gamma(\theta)}{k}}\right)
\end{gathered}
$$

where $k$ is the number of bits per symbol and $\gamma(\theta)$ is the signal-to-noise ratio (SNR), which is affected by the array factor given by:

$$
\gamma(\theta)=\frac{\mathrm{SNR}}{N}\left|\frac{\sin \left(\frac{N k_{0} d \sin \theta}{2}\right)}{\sin \left(\frac{k_{0} d \sin \theta}{2}\right)}\right|
$$

where $N$ is the number of antennas, $k_{0}=\frac{2 \pi f_{c}}{c}$ the free space wave number, $f_{c}$ the carrier frequency, $c$ the light velocity, $d$ the distance between adjacent antennas, and SNR is fixed for $\theta=0^{\circ}$. It is to be noted that the BER in the broadside direction does not change with the number of antennas considered in the array because of the normalization chosen in (3).

Fig. 2 shows the results obtained with $N=7, d=0.8 \lambda_{c}$ ( $\lambda_{c}$ is the wavelength at $f_{c}$ ) and SNR $=20 \mathrm{~dB}$. The array factor shows that the $-3 \mathrm{~dB}$ beamwidth is about $9^{\circ}$. By arbitrarily fixing a threshold of received uncoded BER at $10^{-3}$ for the user to be able to retrieve the data, the QPSK modulation leads to a beamwidth of $17.1^{\circ}$ while the 16-QAM leads to $13.2^{\circ}$, this last one being more sensitive to noise. 


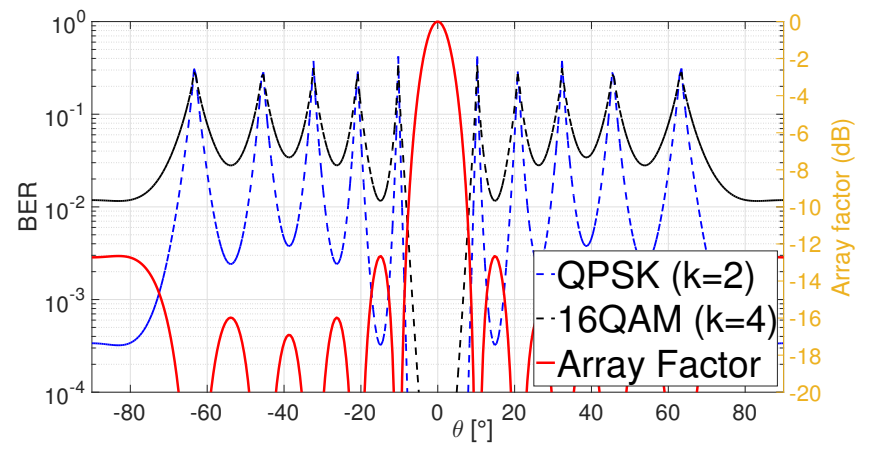

Fig. 2. 7-antenna array: array factor and BER for QPSK and 16-QAM.

\section{Spatial Data Focusing Scenario}

The scheme proposed in this paper is illustrated in Fig. 3 The data symbols to be transmitted are mapped onto a $N$ orthogonal-dimension space, and each dimension is then modulated and transmitted separately on a unique antenna. In doing so, the signals $x_{n}(t)$ are not correlated and the power gain due to the array factor is lost. However, the data focusing is largely increased. When the user moves away from the broadside direction (or any desired direction if a phase/delay law is used), the orthogonality between dimensions is not preserved anymore and the $\mathrm{N}$-dimension constellation is severely deteriorated. The angular rate at which the orthogonality is lost depends on multiple parameters, including the nature of the dimensions as well as the considered mapping.

To illustrate this approach and to quantify the focusing performance, we consider $N$ dimensions (hence $N$ antennas) that are orthogonal in time domain. Each dimension is represented by a raised cosine pulse. If $f_{d}$ is the symbol rate, each pulse duration is $\frac{1}{N f_{d}}$. For the sake of clarity, only a Pulse Amplitude Modulation is used here. Simulations are performed using Matlab. A stream of $10^{5}$ random bits is generated and symbols of $k$ bits are mapped onto the $N$-dimension space. BER results are shown in Fig. 4 (simulation parameters are given in the caption). When $N=2$, the beamwidth observed at $\mathrm{BER}=10^{-3}$ is $29.4^{\circ}$ when $k=2$ and $12.8^{\circ}$ when $k=4$, which is similar to the power focusing with $N=7$ and 16-QAM. The SDF scheme is therefore interesting since it can achieve the same data focusing performance with a much smaller array. When $N=7, \mathrm{SDF}$ achieves a beamwidth of $5^{\circ}$ when $k=7$ and $2.5^{\circ}$ when $k=14$. For a fair comparison, i.e., similar robustness to noise, we compare here $k=2$ and $k=4$ in two dimensions to $k=7$ and $k=14$ in seven dimensions, respectively. In other words, whatever $N$, two cases are compared: $k=N$ and $k=2 N$. It is to be noted that when $N=7$, the symbols contain more bits so the bit rate is increased. However, to create multiple dimensions, more bandwidth is required. So as with classical multidimensional modulations, the spectral efficiency remains unchanged when $N$ varies and the overall bandwidth is fixed. Hence SDF may use any number of antennas while keeping constant the bitrate and the robustness to noise.

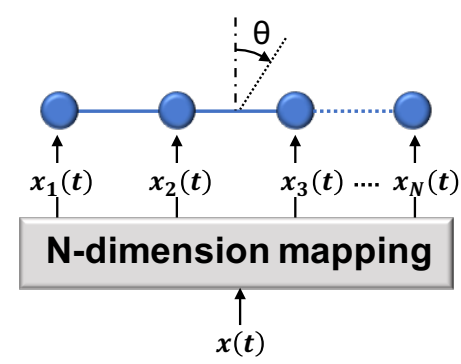

Fig. 3. Spatial Data Focusing (SDF) scheme using $N$ dimensions and $N$ TX antennas.

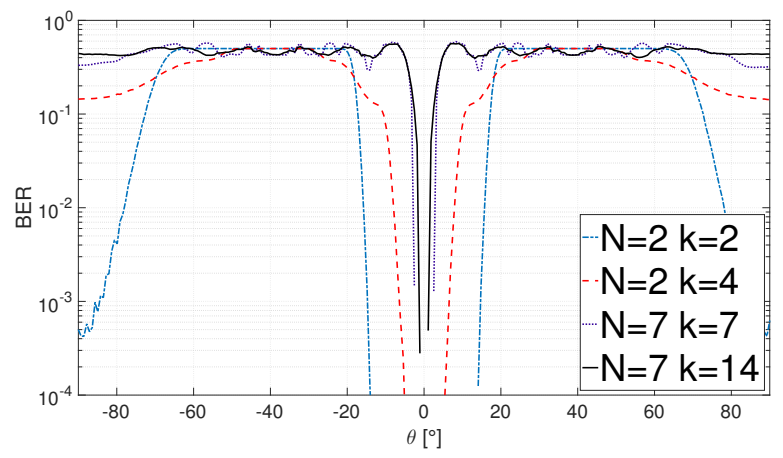

Fig. 4. BER obtained with a N-antenna array using Spatial Data Focusing scheme. $d=0.8 \lambda_{c}, f_{d}=20 \mathrm{MHz}$, roll-off $=0.75$, SNR $=20 \mathrm{~dB}$.

\section{CONCLUSION AND PERSPECTIVES}

By focusing the data instead of the power, the scheme introduced in this paper can overcome beamwidth limitations of classical antenna arrays in wireless geocasting scenarios. When the user is not located within a predetermined beam with respect to the multiple-antenna base station, the received signal is deteriorated as the orthogonality of the constellation is not preserved. This is somehow similar to Directional Modulation (DM) techniques. However, in DM techniques such as in [3], the goal is to keep the antenna array radiation unchanged while deteriorating the constellation outside the main lobe direction, thereby achieving both power and data focusing. However, the beam within which the received BER is low is about the same size than the $-3 \mathrm{~dB}$ beamwidth. By releasing the power focusing constraint, SDF achieves much narrower beams, at the expense of a smaller power efficiency.

This work was supported by the ANR GEOHYPE project, grant ANR-16-CE25-0003 of the French Agence Nationale de la Recherche.

\section{REFERENCES}

[1] T. Mavridis, J. Sarrazin, L. Petrillo, P. D. Doncker, and A. Benlarbi-Dela, "Information spatial focusing scheme for uwb wireless communications in smart environments," IEEE Antennas and Wireless Propagation Letters, vol. 14, pp. 20-23, 2015.

[2] K. Cho and D. Yoon, "On the general ber expression of one- and twodimensional amplitude modulations," IEEE Transactions on Communications, vol. 50, no. 7, pp. 1074-1080, Jul 2002.

[3] Y. Ding and V. F. Fusco, "Constraining directional modulation transmitter radiation patterns," IET Microwaves, Antennas Propagation, vol. 8, no. 15 , pp. $1408-1415,2014$. 